\title{
The Fog Computing Service for Healthcare
}

\author{
YingJuan Shi \\ Mathematics, Physics and Information Engineering \\ Zhejiang Normal University \\ Jinhua, China \\ Email:tea_shi@163.com
}

\author{
Hui Wang \\ Mathematics, Physics and Information Engineering \\ Zhejiang Normal University \\ Jinhua, China \\ Email:hwang@zjnu.cn
}

\author{
Si Lu \\ Zonesion (Wuhan) Technology Co., Ltd. \\ Wuhan, China \\ Email: lusi@zonesion.com.cn
}

\begin{abstract}
Fog Computing is a new architecture to migrate some data center's tasks to the edge of the server. The fog computing, built on the edge servers, is viewed as a novel architecture that provides the limited computing, storing, and networking services in the distributed way between end devices and the traditional cloud computing Data Centers. It provides the logical intelligence to the end devices and filters the data for Data Centers. The primary objective of fog computing is to ensure the low and predictable latency in the latency-sensitive of Internet of Things (IoT) applications such as the healthcare services. This paper discusses the characteristics of fog computing and services that fog computing can provide in the healthcare system and its prospect.
\end{abstract}

Keywords-fog computing; IoT; cloud computing; latency; healthcare

\section{INTRODUCTION}

With the development of the Internet of Things (IoT), a growing number of sensors have been employed, leading to an increasing amount of data generated by them. To explore the intelligence of data, the Cloud Computing has been used to perform the storage, processing and data analysis. The "payas-you-go" cloud computing model provide an efficient alternative to own and manage private data centers (DCs) for customers facing Web applications and batch processing. Therefore, the cloud computing frees the enterprise and the end users from the specification of many details [1].

Since computing devices assess the environment by interacting with networking devices, one of fundamental global properties of the network is connectivity, which ensures that all devices can communicate with each other over the single hop or multiple hops. The connected sensors distribute the generated data to central storing and computing devices, called the Cloud. However, the collected task of the Cloud are rather difficult to be executed. The reasons are as follows:

\author{
GeJian Ding \\ Institute of Continuing Education \\ Zhejiang Normal University \\ Jinhua, China \\ Email:dgj@zjnu.cn
}

\author{
H. Eduardo Roman \\ Department of Physics, University of Milano-Bicocca, \\ Piazza della Scienza 3, 20126 Milan (Italy) \\ Email: eduardo.roman@mib.infn.it
}

1) When the Cloud is relatively far from the customer, it becomes a problem for the latency-sensitive applications. In order to reduce the operating costs of cloud computing, cloud server providers prefer to build data centers in places with the low-cost resources. These places are generally in the remote areas, and therefore will increase the data transmission delay. For example, we found that it takes about one second to get the data generated by temperature and humidity sensors but stored in the Cloud in our experiments. When it comes to the delay-sensitive applications, such as body area networks and vehicle networks, the delay is generally within milliseconds or even microseconds. Therefore, the latency is a big problem worthy of attention.

Furthermore, compared to the transmission delay, the calculation delays shouldn't be ignored as well. As more and more equipment and technology of IoT involved in our daily lives, data calculations requires some time to handle the massive data.

2) With the development of IoT, the amount of data and data nodes keep increasing, resulting in inevitably the increasing burden on the cloud. As a complex cyber-physical system, the IoT integrates all kinds of sensing, identification, communication, networking and information management devices into a system, which link people and things based on the cloud, so that information can be sent and received immediately [2]. Since the data generated by the IoT to the cloud is huge, it not only takes up a lot of network bandwidth, but increases the burden on the data center. As a result, data transmission and information distribution become an issue.

The Cloud cannot meet all the requirements of QoS (Quality of Service) in IoT, so that a new architecture is needed. The new architecture requires the quick reaction to the underlying device, and can reduce the burden on the cloud. In considering how to make the device itself or an "intermediate 
device" to store and handle massive amounts of data generated by the IoT, the fog computing is a good candidate. The main idea of fog computing is to migrate some data centers' tasks to fog nodes on the edge of the server. Thus, it has the advantage of high data transfer rate, thereby reducing user response time.

The advent of fog computing has advanced into a technological revolution providing profitable opportunities for several domains such as body area network(BAN), vehicle networks, and smart grid. The rest of paper is organized as follows: Section 2 discusses what fog computing is and its characteristics. Section 3 describes the services the fog computing can provide. Section 4 describes the future role the fog computing is expected to play. Section 5 is the conclusion.

\section{Fog COMPUTING}

In order to solve the above problems, Cisco proposed the fog computing. Simply, the Fog is a Cloud close to the "ground", actually close to the terminal equipments.

\section{A. What is fog computing}

The fog computing, built on the edge servers, is regarded as a novel architecture that provides the limited computing, storing, and networking services in the distributed way between end devices and the traditional cloud computing DCs. It provides the logical intelligence to the end devices and filters the data for DCs, with the primary objectives in mind: low and predictable latency in the latency-sensitive IoT applications, particularly in the healthcare area.

\section{B. The Implemented Fog}

A simple fog computing architecture is shown in Fig. 1. In this framework, the Fog is between the end devices and the Cloud, with each smart thing attached to one of the fog nodes.

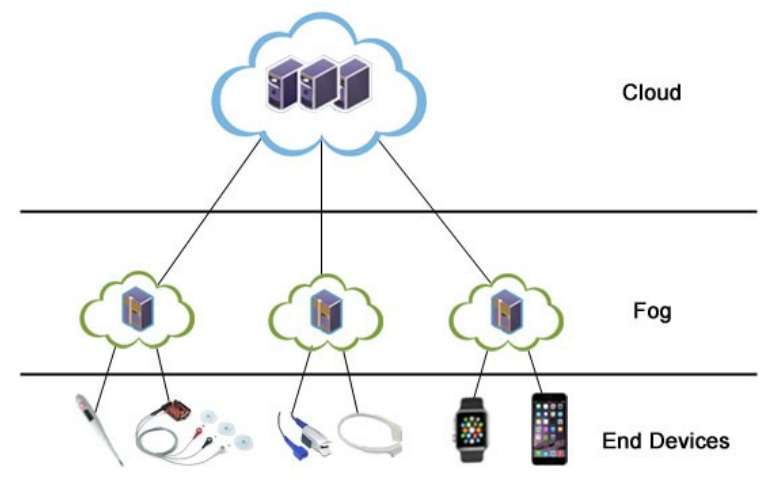

Fig. 1. Fog Computing architecture

Fog nodes, consisting of dispersed devices with weak performance which are deployed at the edge of network, perform the tasks such as scheduling the storing and networking resources and managing distributed computation. These devices are called the "Fog" in this paper. As shown in Fig. 2, the Fog are heterogeneous in nature, ranging from the high-end servers, edge routers, access points, set-top boxes, to the end devices, such as mobile phones, apple watch, and sensors etc [3].

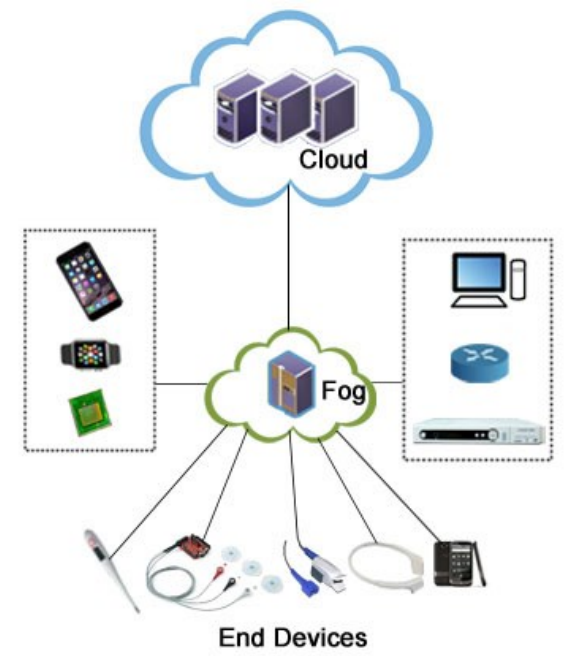

Fig. 2. Edge Servers

\section{Characteristics of the Implemented Fog Computing}

The implemented fog computing extends the cloud computing paradigm to the edge of the network [3]. Both Cloud and Fog provide storage, computation, and network services to end-users. However, the Fog is different from the Cloud in terms of the networking, computing, storing task, the QoS as well as its physical location. The fog computing, as a new architecture, implies a number of characteristics that makes the Fog a non-trivial extension of the Cloud.

\section{1) Adjacent physical location}

The Fog is much closer to the "ground". As shown in Fig. 1 , the "cloud computing" can be regarded as the floating sink point at a distant place in the sky, while the "fog computing" can be deployed locally as the neighboring sink point. Since the Cloud is far away from the end devices, the end devices are connected to the Cloud through various routers and networks. Therefore, the bottleneck of resources in networks will inevitably lead to unpredictable delays. As the amount of sensitive processed information increases, critical and latencysensitive data cannot be handled in time, eventually causing serious problems.

As the fog nodes are deployed at the network edge, they can greatly reduce the time delay. As shown in Fig. 3, if the end devices are in the same Local Area Network (LAN), transmission delay can be reduced owing to available networking resources compared to the Wide Area Networks (WANs) or Internet. When various and dynamic generated data reach the Fog, they are automatically stored and processed depending upon their type and urgency level. In a word, the Fog can reduce the overall delay by exchanging the information within the LAN. 


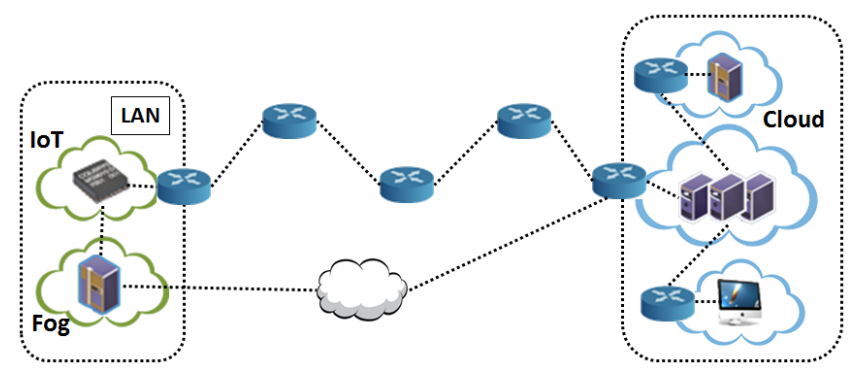

Fig. 3. Location of IoT between fog and cloud

\section{2) Support for on-line analytics}

The Fog is deployed to play a significant role in the storing and computing of the data close to the end devices. Since the Fog is a storage and calculation place around end devices, it can provide real-time analysis for them and, furthermore, give the appropriate response actions to them.

The Fogs provide distributed local storage based on their memory. Based on the rapid development of Bigtable, HBase, NoSQL and other open-source, distributed and non-relational modeled databases, cloud computing can support massive data storage. Instead, the Fog is only needed to support the storage of a small amount of data. The data maybe include the wide range of monitoring data, meta data, computing strategy and etc. Because of the diversity of data, the Fog must support different types of storage strategies, e.g., ephemeral storage for monitoring data or semi-permanent at the higher tier [3].

According to various QoS, while the Cloud provides parallel computing and intelligence analysis for big data through multiple servers, the Fog implements limited computing in order to achieve dynamic and real-time online analysis. The fog nodes play a significant role in the data collection and data processing [1].

\section{3) Service is provid by smart but not powerful devices}

The fog nodes only perform limited computing. Thus, they do not need to use powerful computers, and only need to use routers, set-top boxes, access points, mobile phones and other cheap end devices. Then, the work of data mining and longterm analysis could be assigned to the distant Cloud. From the physical point of view, the traditional Cloud is driven by the servers whereas the Fog can be driven by the existing "smart devices" around us, like personal computers, phones, gateways, etc.

\section{4) Support for various communications networks}

The end devices come in different form of factors, and will be supported for a wide variety of communication networks. In the IoT, there are large-scale sensor networks to monitor the environment. When different sensors connect to the Internet, different types of protocols are involved. As shown in Fig. 4, some of the protocols are supported by the Fog, such as ZigBee, WiFi, 2G/3G/4G, WiMax, 6Lowpan and so on. However, the network supported by the Cloud is the single one, which is the TCP/IP protocol.

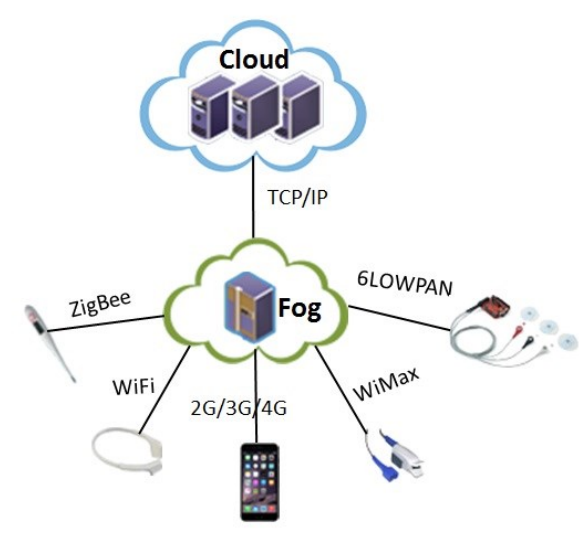

Fig. 4. Protocol support

\section{5) Distributed Computing}

The fog computing is working in the distributed way, rather than the centralized way. For the Fog, to provide a service, the enormous computation effort is required to be decomposed into many smaller parts, and then many of them are assigned to the fog nodes for processing. In contrast, the Cloud puts these calculation results together or undertakes a much more complex calculation as the final result.

As the amount of data increases and the heterogeneity as well as the unpredictability come into play, the bandwidth will become an issue in addition to delays. Since a fog node in fog computing is responsible for dealing with the regional data, such the distributed computing strategy helps to reduce the computational complexity, resulting in the raised calculation rate. Finally, the computed data, relayed by the fog nodes, were continuously uploaded to the Cloud, which is beneficial to solve the bandwidth issue.

\section{THE DUTY OF Fog COMPUTING}

The key characteristics of the fog computing have been discussed in the previous section, a platform to deliver a rich portfolio of new services and applications at the edge of the network is described in this section. The Fog is constructed as an unifying platform, to deliver the new breed of emerging services and enable the development of new applications.

In this fog model in Fig. 5, all the local devices and sensors deployed at the edge attach to the local Fog, which connect to the Cloud. The generated data are transmitted to the fog nodes. The Fog would do some processing after receiving these data, and then forward to the Cloud. The Cloud mainly does the big data storage and data mining, and feedbacks some calculation results to the Fog. The Fog would give the commands to the end devices in order to control them according to the calculation results. Here, the fog nodes can be used in a similar but more powerful way than the gateway equipments.

In summary, the Fog focuses on the following jobs: Switching Network, Pushing Service and Core Service. 


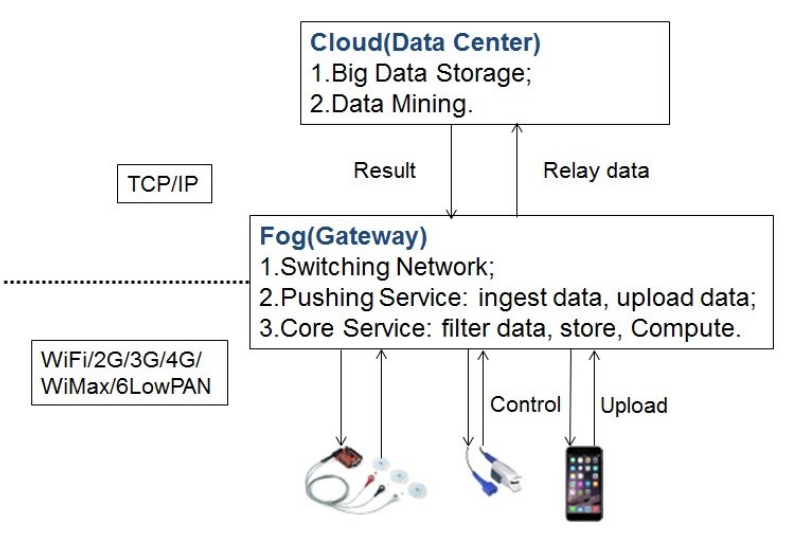

Fig. 5. Fog Computing architecture with specific duty

\section{A. Switching Network}

As previously mentioned, the Fog needs to support many protocols, such as ZigBee, WiFi, 2G/3G/4G, WiMax, 6LOWPAN and so on. However, the Cloud only supports TCP/IP. As far as we know, different communication protocols transfer data in the different formats. Then, the first thing the Fog should do is to switch network between IoT and Cloud.

\section{B. Pushing Service}

The Fog as an intermediary between the end devices and the Cloud needs to provide pushing service to both of them. The pushing service includes receiving the data and uploading the data. The end devices upload data to the Fog after they generate the data. Then, the Fog should ingest these data and update processed data to Cloud for long-time storage and deeply digging.

\section{Core Service}

The two functions mentioned above are the basic to support the Fog to do some storage and computing jobs. The Fog's main duty is to achieve the local data processing, storing and computing in the devices with the weak performance.

When a device is connected to the Internet and generate data, there is a possibility that at some stage it is no longer necessary to upload the data to the cloud [4]. For this purpose, the Fog, connecting IoT to the cloud, should be having the extra functionality to do some processing like filtering and aggregation before sending them to the Internet and eventually to the Cloud. The Fog must decide what it should send (content), how to send it (data format), and when to send it (time). In this process, it also need to delete some redundant or invalid data, and aggregate the complementary and redundant data in the space and time dimensions.

Filtering and aggregating data need some policies, and online analysis needs some real or related data. The Fog should support ephemeral and semi-permanent storage [5]. There are several problems should be tackled, such as which data to be stored at network edge and how to store data optimally with the limited storage capacity.
The Fog calculates the local data to realize the intelligent on-line analytics. The Fog only needs to do some calculation for the application which requires high real-time, such as the healthcare services. The intelligent computing based on the Fog is a complex task, because different applications have different calculation requirements.

\section{THE ROLE OF Fog COMPUTING}

As discussed earlier, the fog computing provides computing, storing, and networking services between the end devices and the traditional Cloud. It performs logical analysis to the end devices, and filters data for the DCs.

\section{A. Its role in the IoT}

The fog computing would collect and process vast amounts of data generated by the end devices, providing online analysis and local decision for the current environment.

The end devices keep collecting data in order to monitor the environment. Accordingly, the fog nodes need to ingest real-time data from the end nodes. In some applications such as body area networks, body sensors would upload a large amount of data frequently. The fog nodes need to do some processing of the data, such as data filtering, data aggregation, etc. For example, if the ECG sensors upload urgent data, the fog node will handle them immediately according to the local policy. Fig. 6 reveals the processes in a simple way.

Because the Fog can support the local data storage, it will be able to do some calculations and intelligent analysis of these data, distribute some decisions based on the policy to the current environment to provide a better service.

\section{B. Its role in the Data Center}

When a device is connected to the Internet and generate data, there is a possibility that at some stage it is no longer necessary to upload the data to the Cloud or sync device due to the existing redundant data [4]

With the fog model, the Cloud retains its central "thinktank" role (analyzing data and making all the global decisions) However, the Cloud can also assign the part of tasks to the fog nodes as it often makes more sense to the localized analysis and the decision-making at the edge. Also, if the Fog don't need to undertake in-depth analytics, they just actively filter the local data and selectively relay the data to the Cloud [6]. Therefore, for the widely distributed sensor networks, the transmission efficiency can be enhanced considerably. 


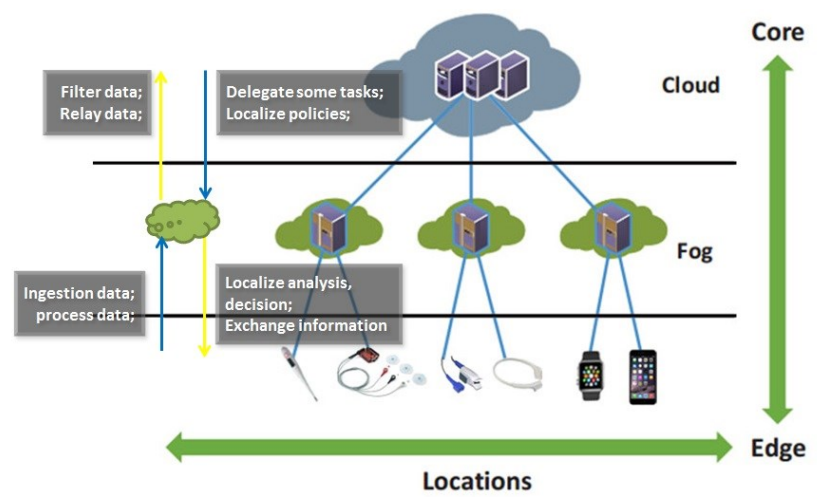

Fig. 6. Fog Computing architecture with specific roles

\section{CONCLUSIONS AND FUTURE WORKS}

In this paper, the vision and the key characteristics of the fog computing is discussed. The platform provides the limited computing, storing, and networking services in the distributed way to resolve the latency issue caused by the IoT and to collaborate with the Cloud. The Fog as an unifying architecture is to execute part of data center's tasks at the edge of the server and, finally, enable the new development of the latency-sensitive IoT applications.

In future works, the local storing and computing strategies, implementing the fog computing in the BAN, vehicle networks and other fields will be studied.

\section{References}

[1] F.Bonomi, R.Milito, J.Zhu, andS.Addepalli. Fog computing and its role in the internet of things[C]. MCC Workshop on Mobile Cloud Computing,2012:13-16.

[2] Zhibo Pang, Lirong Zheng, etc. Design of a terminal solution for integration of in-home health care devices and services towards the Internet-of-Things[M]. TAYLOR \& FRANCIS LTD Publishing,2015:310.

[3] JanuszKacprzyk, Warsaw, Poland. Big Data and Internet of Things: A Roadmap for Smart Environments[M]. Switzerland: Springer International Publishing,2014:168-185.

[4] AazamM, HungP, HuhE. Smart gateway based communication for cloud ofthings[C].IEEE,2014:1-6.

[5] Ivan Stojmenovic, Sheng Wen. The Fog Computing Paradigm: Scenarios and Security Issues[C]. Federated Conference on Computer Science and Information Systems, 2014:1-8.

[6] Fog Computing — clearly the way forward for IoT[Online]. Available: http://blog.opengear.com/fog-computing-clearly-the-way-forward-foriot. 